\title{
Practical Management of Well Differentiated Thyroid Carcinoma in Korea
}

\author{
Yong SAng LeE, Kee-Hyun NAM, Woong Youn CHUnG, HANg Seok CHANG, NAOYuKi SHIGEMATSU*, \\ HIROSHI TAKAMI**, ATSUSHI KUBO* AND CHEONG SOO PARK \\ Department of Surgery, Yonsei University College of Medicine, Seoul, Korea \\ *Department of Radiology, Keio University School of Medicine, Tokyo, Japan \\ **Department of Surgery, Teikyo University School of Medicine, Tokyo, Japan
}

\begin{abstract}
Objectives: The optimal extent of surgery and postoperative management of patients with well differentiated thyroid carcinoma (WDTC) vary among countries and institutions. We assessed the practical management of WDTC in Korea by questionnaire and compared these results with those obtained in similar surveys of members of the Japanese Society of Thyroid Surgery (JSTS) and the International Association of Endocrine Surgeons (IAES). Materials and Methods: Questionnaires were sent by mail or e-mail to 266 members of the Korean Association of Endocrine Surgeons (KAES). Ninety members (33.8\%) completed the questionnaire; their responses were compared with those of the JSTS and IAES surveys. Results: Total thyroidectomy was more prevalent in the KAES and IAES than in the JSTS, irrespective of tumor size in the low-risk group. Patients with papillary microcarcinoma were more likely to undergo aggressive central compartment node dissection in the KAES than in the IAES or JSTS. Thyroid stimulating hormone suppression therapy was administered to a higher proportion of patients and for longer times in the KAES and IAES than in the JSTS. Postoperative radioactive iodine treatment was more prevalent in the KAES than in the JSTS. There were no differences between the KAES and the JSTS in the treatment of patients with locally advanced thyroid carcinoma. External irradiation and radioactive iodine treatment for recurrent papillary thyroid carcinoma were favored more by the KAES than the IAES and JSTS. Conclusions: The actual practices of members of the KAES were almost similar to those of the IAES, but differed from those in Japan in some aspects. In general, however, members of the KAES favored more aggressive treatment of WDTC than did physicians in other countries.
\end{abstract}

Key words: Well differentiated thyroid carcinoma, Practical management, Questionnaire

(Endocrine Journal 55: 1015-1024, 2008)

THE principal treatment for patients with well differentiated thyroid carcinoma (WDTC) is surgical resection. However, the practical management of patients with WDTC has been found to vary among nations and institutions, including the extent of thyroid resection and lymph node dissection, the use of postoperative radioactive iodine (RAI) treatment and thyroid stimulating hormone (TSH) suppression therapy, and the treatment of patients with locally advanced unre-

Received: July 18, 2008

Accepted: July 28, 2008

Correspondence to: Cheong SOO PARK, M.D., Ph.D., Department of Surgery, Yonsei University College of Medicine, 250 Seongsanno, Seodaemun-gu, Seoul 120-752, Korea sectable thyroid carcinoma, and those with recurrent thyroid carcinoma.

To determine the practical management of WDTC in Korea, we distributed a questionnaire survey [1], to members of the Korean Association of Endocrine Surgeons (KAES). These findings were compared with the results of similar surveys of members of the Japanese Society of Thyroid Surgery (JSTS) and the International Association of Endocrine Surgeons (IAES) $[1,2]$.

\section{Materials and Methods}

Questionnaires in Korea, adapted from the question- 
naire sent to members of the JSTS [1], were sent by mail or e-mail to 266 members of the KAES. The questionnaire consists of five case descriptions and questions related to each. The presented cases were; low-risk papillary thyroid carcinoma smaller than $2 \mathrm{~cm}$ (T1); low-risk papillary thyroid carcinoma larger than $2 \mathrm{~cm}$ (T2); high-risk poorly differentiated papillary thyroid carcinoma; high-risk locally advanced thyroid carcinoma; and recurrent papillary thyroid carcinoma. The full text of the questionnaire has been published [1].

Of the 266 members of the KAES sent questionnaires, $90(33.8 \%)$ responded. Among the 90 respondents, $61(75.3 \%)$ were employed by university hospitals, $12(14.8 \%)$ by general hospitals, and $8(9.9 \%)$ by private clinics. Stratified by district, respondents included 30 respondents (37\%) from Seoul, 19 (23.5\%) from Gyeonggi-do, 3 (3.7\%) from Gangwondo, 5 (6.2\%) from Chungcheong-do, 13 (16.0\%) from Gyeongsang-do, and 11 (13.6\%) from Jeolla-do, reflecting an even distribution throughout Korea.

\section{Results}

Treatment modalities for small papillary carcinomas of the thyroid (T1)

\section{$\checkmark$ Case presentation}

An ultrasound examination performed during a routine medical checkup detected a thyroid adenoma in a 35-year-old woman, who sought further examination in the department of surgery at a leading hospital in her district. An aspiration cytology under ultrasonic guidance showed papillary carcinoma, while the ultrasonic imaging revealed that it was localized at the inferior pole of the thyroid lobe with no signs of tumor infiltration. No enlarged lymph nodes were observed.

Almost all Korean respondents reported they would perform fine-needle aspiration cytology (FNAC) for small thyroid nodules, irrespective of tumor size. Of the Japanese respondents, $40 \%$ reported they would perform FNAC for tumors $0.5 \mathrm{~cm}$ or smaller, $82 \%$ for tumors $0.5-1.0 \mathrm{~cm}$, and $100 \%$ for tumors $1.0-2.0 \mathrm{~cm}$ in size. If cytology was positive, most of the KAES and IAES respondents favored surgical treatment irrespective of tumor size. Only $27 \%$ of Japanese respondents, however, recommended surgery for patients with microcarcinomas, particularly for tumors smaller than $0.5 \mathrm{~cm}$ in diameter (Table 1).

Most respondents from the KAES and IAES recommended less-than-total thyroidectomy or total thyroidectomy for patients with tumors $0.5 \mathrm{~cm}$ or smaller, and $0.5-1.0 \mathrm{~cm}$ in diameter, and total thyroidectomy for patients with tumors lager than $1.0 \mathrm{~cm}$ in size. Japanese respondents, however, preferred less-than-total thyroidectomy (Fig. 1).

Compared with members of the JSTS and IAES, a higher percentage of members of the KAES recommended lymph node dissection. Japanese respondents showed a greater preference for bilateral neck dissection than did the Korean and IAES respondents (Fig. 2).

Treatment modalities for large papillary carcinomas of the thyroid (T2, low-risk group)

\footnotetext{
- Case presentation

A 40-year-old man presented to the department of surgery of a leading hospital in his district with a complaint of a mass detected at the right thyroid lobe. Ultrasound and CT examinations revealed a mass $3 \mathrm{~cm}$ in diameter, localized within the thyroid gland and 2 enlarged lymph nodes each on the paratracheal and the lateral side of the internal jugular vein of the affected site. A cytological examination yielded a well-differentiated papillary carcinoma of the thyroid. Laryngoscopy exhibited normal movement of the vocal cords. Neither CT, nor ${ }^{99 \mathrm{~m}} \mathrm{Tc}$ bone scanning revealed evidence of distant metastasis.
}

All of the respondents in the KAES and IAES

Table 1. Surgery performed for small papillary thyroid carcinomas (T1) stratified by primary tumor size (T1, low-risk group)

\begin{tabular}{lccrrr}
\hline \multirow{2}{*}{ Tumor size } & \multirow{2}{*}{ Korea } & Japan & & \multicolumn{3}{c}{ IAES } \\
\cline { 4 - 6 } & & & Europe & Asia/Oceania & America \\
\hline $0-0.5 \mathrm{~cm}$ & $92.6 \%$ & $27 \%$ & $97 \%$ & $100 \%$ & $100 \%$ \\
$0.5-1.0 \mathrm{~cm}$ & $100 \%$ & $60 \%$ & $97 \%$ & $100 \%$ & $100 \%$ \\
$1.0-2.0 \mathrm{~cm}$ & $100 \%$ & $99 \%$ & $100 \%$ & $100 \%$ & $100 \%$ \\
\hline
\end{tabular}



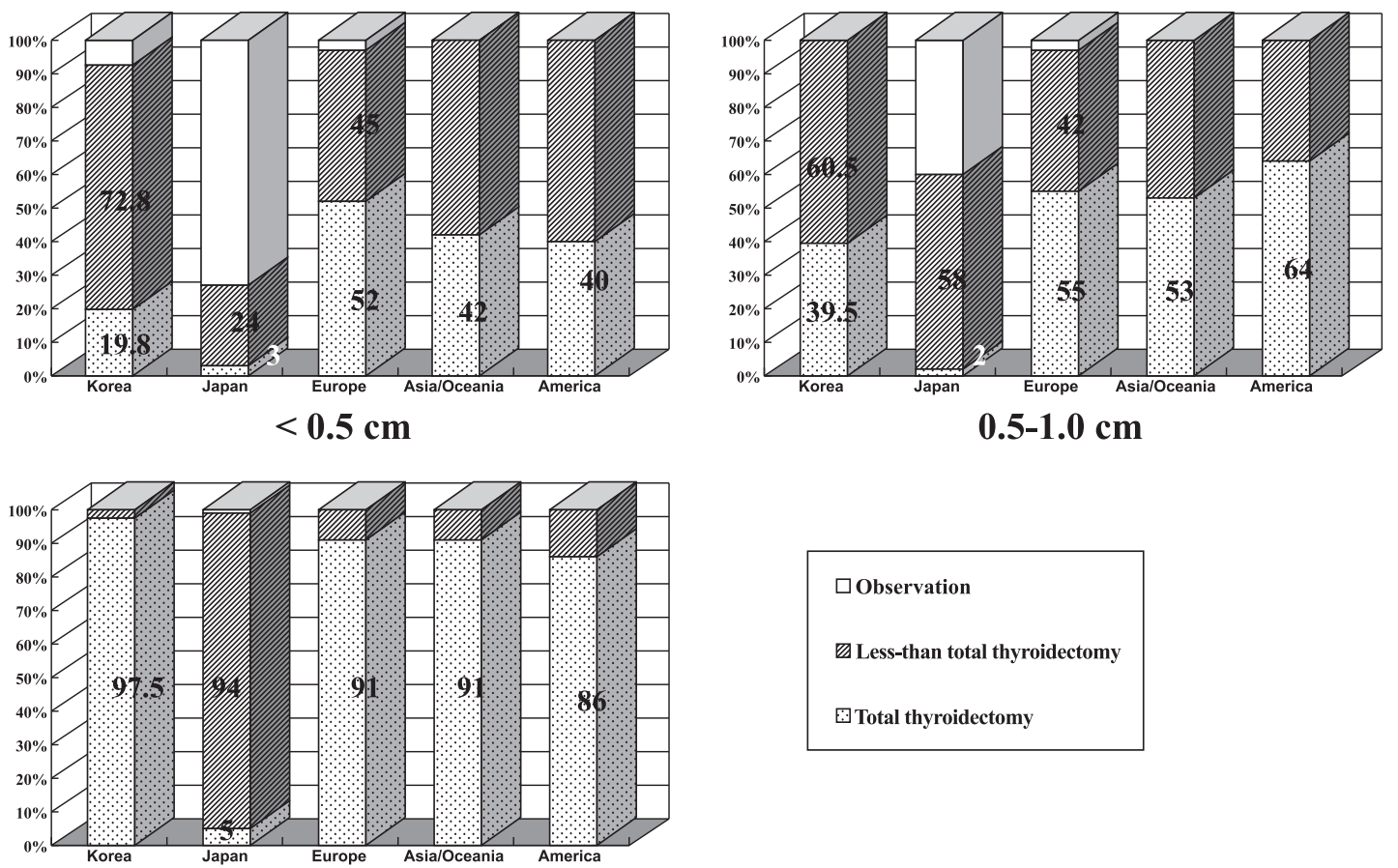

\section{$1.0-2.0 \mathrm{~cm}$}

Fig. 1. Extent of thyroidectomy stratified by primary tumor size in small papillary thyroid carcinoma (T1, low-risk group).
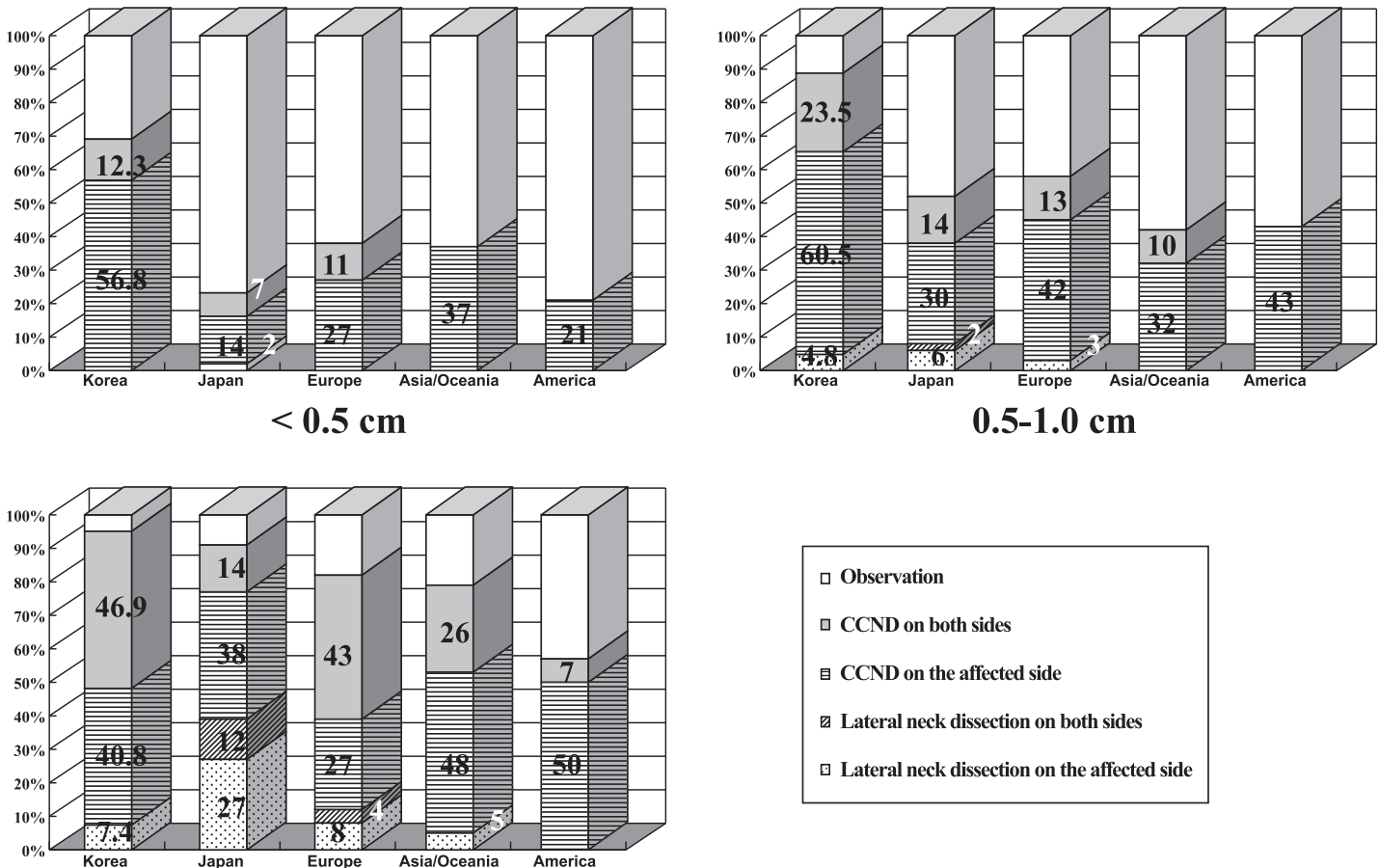

$1.0-2.0 \mathrm{~cm}$

Fig. 2. Extent of cervical lymph node dissection for small papillary thyroid carcinoma stratified by primary tumor size (T1, low-risk group) 


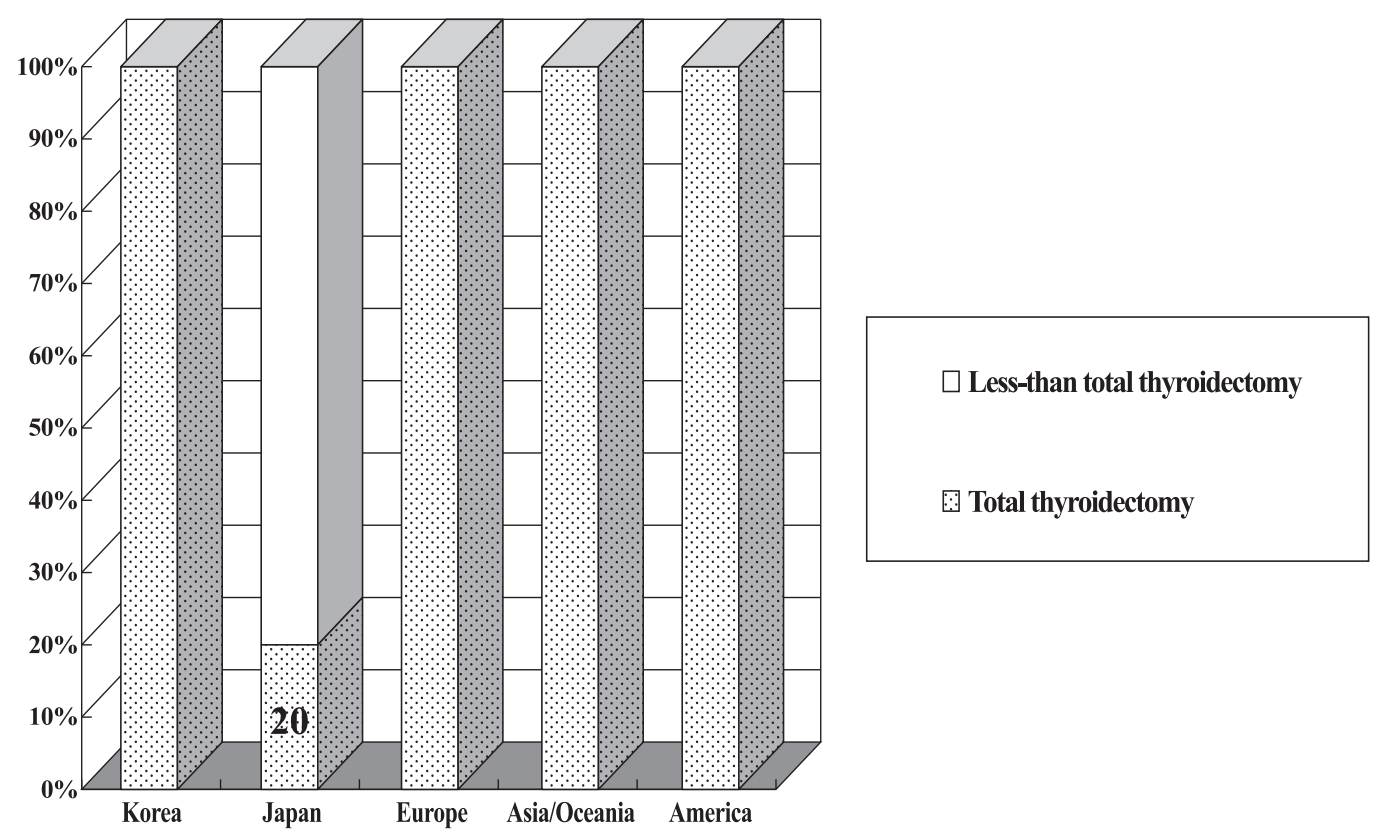

Fig. 3. Extent of thyroid resection in large papillary thyroid carcinoma (T2, low-risk group).

Table 2. Extent of lymph node dissection for large papillary thyroid carcinoma (T2, low-risk group)

\begin{tabular}{lrrrrr}
\hline \multirow{2}{*}{ Extent of node dissection } & \multirow{2}{*}{ Korea } & Japan & \multicolumn{3}{c}{ IAES } \\
\cline { 4 - 6 } & & & Europe & Asia/Oceania & America \\
\hline CCND on the affected side & $98.8 \%$ & $100 \%$ & $97 \%$ & $100 \%$ & $100 \%$ \\
CCND on both sides & $80.2 \%$ & $67 \%$ & $64 \%$ & $53 \%$ & $50 \%$ \\
LND on the affected side & $88.9 \%$ & $93 \%$ & $91 \%$ & $84 \%$ & $93 \%$ \\
LND on both sides & $4.9 \%$ & $11 \%$ & $18 \%$ & $5 \%$ & $7 \%$ \\
\hline
\end{tabular}

* Abbreviations: CCND, central compartment node dissection; LND, lateral neck dissection

surveys preferred total thyroidectomy, compared with $20 \%$ of the JSTS respondents. Less-than-total thyroidectomy was more frequently chosen in Japan (Fig. 3).

The extent of lymph node dissection is shown in Table 2. Dissection of the central compartment and lateral lymph nodes on the affected side was recommended by $98.9 \%$ and $99.9 \%$, respectively, of the Korean respondents, and bilateral dissection of central and lateral lymph nodes by $80.2 \%$ and $4.9 \%$, respectively.

Compared with members of the JSTS and IAES, a higher percentage of members of the KAES recommended contralateral central compartment lymph node dissection, whereas a lower percentage recommended bilateral neck node dissection (Table 2).

All Korean respondents recommended postoperative TSH suppression therapy and most $(96.3 \%)$ would continue administering suppression therapy for life. The results of the IAES questionnaire were similar. In contrast, $37 \%$ of JSTS respondents did not recommend TSH suppression therapy, and only $34 \%$ favored its administration for life (Fig. 4).

Postoperative radioactive iodine (RAI) treatment was recommended by $82.7 \%$ of the KAES respondents and a similar percentage of IAES respondents. In contrast, only $46 \%$ of JSTS respondents recommended postoperative RAI treatment (Table 3).

Treatment modalities for high-risk papillary carcinomas of the thyroid (poorly-differentiated)

\footnotetext{
- Case presentation

On ultrasound and CT scanning, a 60-year-old man was found to have a thyroid tumor measuring $6 \mathrm{~cm}$ in diameter. Extra-thyroidal invasion was observed but there was no evidence
} 


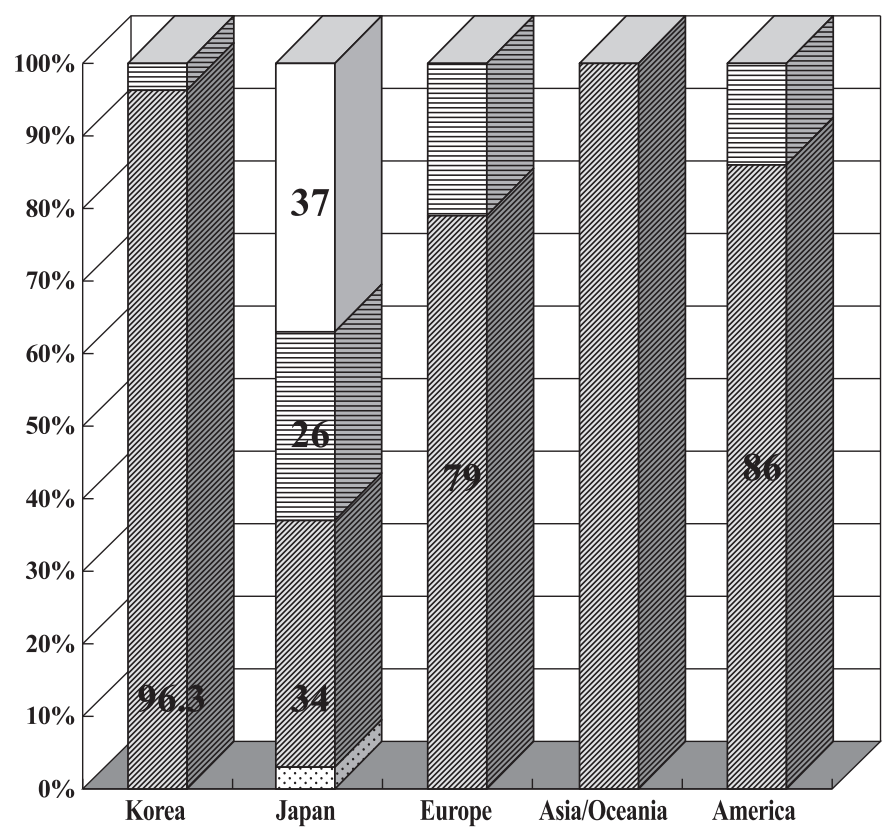

$\square$ Not conducted

目At least 5 years

Throughout lifetime

For 1-2 years

Fig. 4. Frequency of TSH suppression therapy after surgery in large papillary thyroid carcinoma (T2, low-risk group).

Table 3. Postoperative high-dose radioactive iodine therapy for large papillary thyroid carcinoma (T2, lowrisk group)

\begin{tabular}{lccccc}
\hline & \multirow{2}{*}{ Korea } & Japan & \multicolumn{3}{c}{ IAES } \\
\cline { 5 - 6 } & & & Europe & Asia/Oceania & America \\
\hline Adopted & $71.5 \%$ & $28 \%$ & $85 \%$ & $90 \%$ & $79 \%$ \\
Desirable, if available & $11.2 \%$ & $18 \%$ & $6 \%$ & $5 \%$ & $0 \%$ \\
Not adopted & $17.3 \%$ & $54 \%$ & $9 \%$ & $5 \%$ & $21 \%$ \\
\hline
\end{tabular}

of distant metastases. An elevated serum thyroglobulin level (negative thyroglobulin antibody) was noted. A cytological examination revealed poorly differentiated papillary carcinoma, which was considered to be a high-risk cancer, and the patient underwent total (near-total) thyroidectomy. Following surgery, the base level of serum thyroglobulin was reduced to below the detectable threshold.

The results were compared only between the KAES and JSTS respondents. We found that $60.3 \%$ of the KAES respondents and $52 \%$ of the JSTS respondents recommended RAI treatment regardless of serum thyroglobulin concentration (Fig. 5). Only 19\% of the KAES and $20 \%$ of the JSTS respondents recommended RAI treatment for patients with high serum thyroglobulin concentration.
Treatment modalities for high-risk well-differentiated thyroid carcinomas (locally advanced)

\section{- Case presentation}

A 70-year-old man was found to have a thyroid tumor measuring $6 \mathrm{~cm}$ in diameter. Ultrasound and CT scanning revealed that the tumor had infiltrated the larynx and esophagus. If surgery is considered to be the treatment of choice, total laryngectomy and esophageal extirpation appeared unavoidable. A cytological examination found the cancer to be papillary carcinoma. CT scanning of the neck revealed that several lateral cervical lymph nodes on both sides were enlarged. There was no evidence of distant metastasis.

These results were also compared only between the KAES and JSTS respondents. In the treatment of patients with locally advanced thyroid carcinoma, 35.8\% of the KAES respondents recommended surgery alone, $21.0 \%$ recommended external irradiation alone, and 


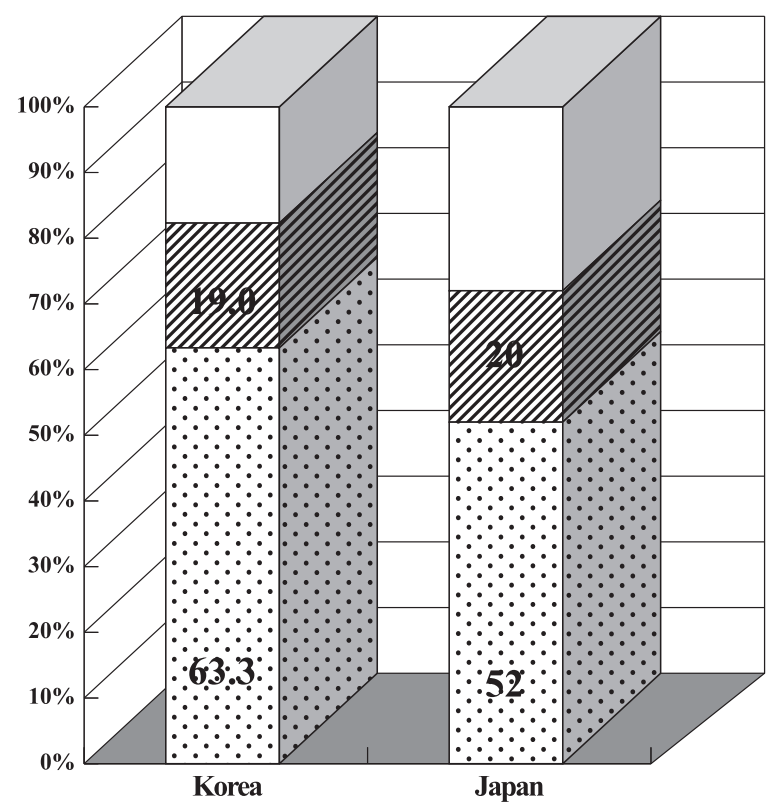

$\square$ No

Yes, if elevated Tg level

$\square$ Yes, regardless of Tg level

Fig. 5. Indication for high-dose radioiodine treatment after total or near-total thyroidectomy for high-risk poorly-differentiated papillary thyroid carcinoma.

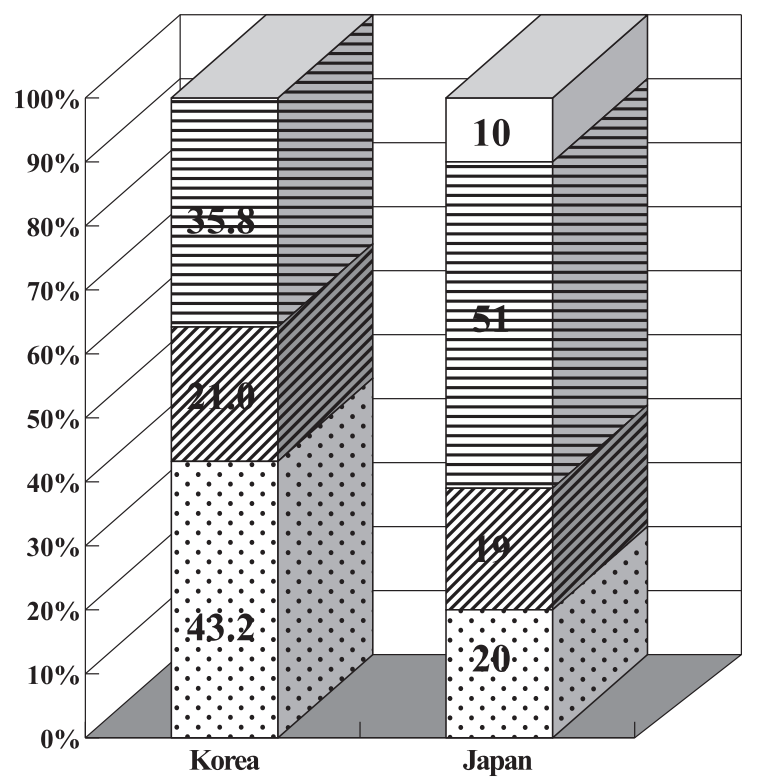

\begin{tabular}{|l|}
$\square$ No treatment \\
$\Xi$ Surgery \\
$\square$ External irradiation \\
$\square$ Surgery + External irradiation
\end{tabular}

Fig. 6. Treatment policies for high-risk well-differentiated thyroid carcinoma (high-risk group, locally advanced).

$43.2 \%$ recommended both.

In contrast, $10 \%$ of the JSTS respondents recommended observation alone, $51 \%$ recommended surgery alone, 19\% recommended external irradiation alone, and $20 \%$ recommenced surgery plus external irradiation $(20 \%)$ (Fig. 6).
Treatment modalities for recurrent papillary thyroid carcinomas after surgery

\section{- Case presentation}

A 60-year-old woman had been subjected to total thyroidectomy and bilateral neck dissection for papillary thyroid carcinoma 5 years previously. Since then, the tumor recurred 
Table 4. Treatment modalities for recurrent papillary thyroid carcinoma

\begin{tabular}{lccccc}
\hline & \multirow{2}{*}{ Korea } & Japan & \multicolumn{3}{c}{ IAES } \\
\cline { 4 - 6 } & & & Europe & Asia/Oceania & America \\
\hline Only observation & $0 \%$ & $2 \%$ & $0 \%$ & $0 \%$ & $7 \%$ \\
Extirpation of metastatic nodes & $70.9 \% *$ & $56 \%$ & $61 \%$ & $58 \%$ & $79 \%$ \\
Modified neck dissection & $6.3 \%$ & $37 \%$ & $36 \%$ & $53 \%$ & $29 \%$ \\
External irradiation & $30.4 \%$ & $14 \%$ & $15 \%$ & $21 \%$ & $0 \%$ \\
PEI treatment & $2.5 \%$ & $5 \%$ & $3 \%$ & $5 \%$ & $0 \%$ \\
High-dose RAI treatment & $95.8 \%$ & $60 \%$ & $73 \%$ & $79 \%$ & $57 \%$ \\
\hline
\end{tabular}

* Berry picking $22.8 \%$, Selective level dissection $48.1 \%$

* Abbreviations: PEI, percutaneous ethanol injection; RAI, radioactive iodine

repeatedly at the cervical lymph nodes and on each occasion, the involved lymph nodes were extirpated. At the latest examination, she was suffering from a fifth recurrence that involved the right cervical lymph nodes.

For recurrent papillary thyroid carcinoma, $2 \%$ of the JSTS and $7 \%$ of the North and South American respondents did not choose any of treatment. Other respondents favored more aggressive treatment modalities.

Overall, the most common treatment modality recommended by the KAES respondents was limited and selective extirpation of the metastatic lymph nodes (70.9\%) rather than radical neck dissection (6.3\%). In contrast, the JSTS and IAES respondents favored wide lymph node dissection.

External irradiation was chosen by $30.4 \%$ of the KAES respondents. Most of the KAES respondents (95.8\%) favored high-dose RAI treatment, as did the JSTS and IAES respondents (Table 4).

\section{Discussion}

According to the new TNM staging system introduced in 2002, the T1 tumors now include all those with diameters up to $2 \mathrm{~cm}$, indicating that the prognosis of patients with these tumors does not differ greatly from that of patients with T1 tumors smaller than $1 \mathrm{~cm}$ in diameter.

In contrast, papillary microcarcinoma defined as a tumor smaller than $1 \mathrm{~cm}$ [3], may show different clinical behaviors than small papillary thyroid carcinoma. Thus, in our questionnaire, T1 tumors subdivided into three groups; smaller than $0.5 \mathrm{~cm}, 0.5-1.0 \mathrm{~cm}$, and $1.0-2.0 \mathrm{~cm}$.
The American Thyroid Association (ATA) guidelines recommended that only thyroid nodules up to $1 \mathrm{~cm}$ in diameter be evaluated by additional invasive diagnostic methods, because only these tumors have the potential to be clinically significant cancers [4]. Furthermore, nonpalpable thyroid nodules have a risk of malignancy equal to that of palpable nodules of the same size [5].

The development of high-resolution ultrasonography and improvements in diagnostic techniques have made it easier to evaluate thyroid nodules. Ultrasonography-guided FNAC is now considered the easiest and most valuable method for assessing thyroid nodules [6].

We found that all KAES and JSTS respondents recommended FNAC for thyroid nodules larger than $1 \mathrm{~cm}$. In contrast, a higher percentage of Korean than Japanese respondents recommended FNAC for thyroid nodules smaller than $1 \mathrm{~cm}$. The difference may be related to overall social and environmental conditions, including patients' desires, accessibility and loyalty to the medical institution, cost of treatment, and fear of cancer.

Both the KAES and IAES respondents recommended total thyroidectomy, regardless of tumor size, for patients with low-risk tumors. In contrast, the JSTS respondents recommended less-than-total thyroidectomy, even for patients with tumors larger than $2 \mathrm{~cm}$ in diameter.

In comparison, both the American Thyroid Association (ATA) and the National Comprehensive Cancer Network (NCCN) recommended total thyroidectomy even for papillary microcarcinomas, for patients $<15$ or $>45$ years of age, a personal history of radiation exposure in the head and neck regions, cervical lymph node or distant metastases, contralateral thyroid nod- 
ules, extra-thyroidal extension, or a first degree family history of well differentiated thyroid carcinoma $[4,5]$. Less-than-total thyroidectomy was regarded as sufficient for low-risk patients with small, isolated, intrathyroidal papillary carcinomas, and an absence of cervical nodal metastases $[4,7]$.

The frequency of recommended lymph node dissection for papillary microcarcinomas was higher in KAES than in the IAES and JSTS respondents, whereas the extent of dissection was equally broader in all three groups as the tumor size increased. The JSTS respondents especially tended to recommend lateral neck node dissection more than the respondents from the other countries.

Overall, total thyroidectomy with central compartment node dissection on the affected side was clearly favored by the KAES and IAES respondents, with the Korean respondents also recommended dissection of the contralateral central compartment nodes. In contrast, the Japanese respondents recommended lessthan-total thyroidectomy along with ipsilateral neck node dissection.

Postoperative RAI treatment was recommended more frequently by the KAES and IAES than by the JSTS respondents. However, because total thyroidectomy is not popular in Japan, JSTS respondents have limited experience with postoperative RAI treatment.

Although RAI treatment is clearly indicated and often beneficial for patients with local or distant residual disease, the use of RAI in low-risk patients remains highly controversial. RAI treatment is less controversial, however, in patients with high-risk papillary thyroid carcinoma [8-11].

Moreover, RAI treatment is generally beneficial following total or near-total thyroidectomy. Thus, the KAES and IAES respondents who had a greater preference for total thyroidectomy, also had a great preference for RAI treatment than the JSTS respondents who preferred less-than-total thyroidectomy.

Life-long TSH suppression therapy was recommended by high percentages of the KAES and IAES respondents. The Japanese respondents, however, either did not recommend. TSH suppression therapy or recommended that it be limited to 5 years.

TSH suppression therapy following thyroidectomy has been reported to reduce the recurrence rate $[12$, 13]. Conversely, it also has been reported to be therapeutically ineffective [14].

Many Korean and Japanese respondents selected high-dose RAI treatment for patients with poorly differentiated thyroid carcinoma. ATA guidelines recommended high-dose (100-200 mCi) RAI treatment for patients suspected or documented to have microscopic residual disease, and those with papillary thyroid carcinoma of aggressive histology (e.g., tall cell, insular, columnar cell variant, etc) [4].

Administration of recombinant human thyroid stimulating hormone (rhTSH) has been reported to stimulate endogenous TSH without the consequences of hypothyroidism [15]. Moreover, successful remnant ablation with low-dose $(30 \mathrm{mCi}) \mathrm{RAI}$ treatment after thyroxine withdrawal was shown to be equivalent to rhTSH stimulation when thyroxine therapy was stopped one day prior to rhTSH injections and restarted the day after radioactive iodine treatment [16].

Serum thyroglobulin concentration measured after TSH stimulation is very useful for detecting recurrence or distant metastasis [17]. More than half of the respondents in Korea and Japan, however, recommended high-dose RAI treatment for patients with poorly differentiated papillary thyroid carcinoma, regardless of serum thyroglobulin concentration.

An overall comparison of recommended treatments for patients with locally advanced thyroid carcinoma shows that Korean respondents favored more aggressive treatments than Japanese respondents. Ten percent of the JSTS respondents selected no treatment. All Korean respondents, however, chose one of the treatment options.

Korean respondents also recommended more aggressive surgical treatment and external irradiation therapy for patients with locally advanced papillary thyroid carcinoma.

Interestingly, Korean respondents preferred including anterior superior mediastinum lymph nodes in the external irradiation field, perhaps due to the tendency of Korean respondents to prefer more aggressive treatment modalities.

Because papillary thyroid carcinomas are relatively insensitive to external irradiation therapy, the treatment outcome may not be improved compared to conventional radiation therapy. It is therefore thought that the methods that can deliver a high dose of radiation to a certain area, such as stereotactic radiation therapy or tomotherapy, may be more beneficial.

Treatment modalities for patients with recurrent papillary thyroid carcinoma, including the extent of metastatic lymph node dissection and the preference 
for high-dose RAI treatment, have been shown to depend on tumor size.

We found that Korean respondents preferred limited and selective extirpation of metastatic lymph nodes over radical neck dissection, whereas the JSTS and IAES respondents preferred radical neck dissection.

Percutaneous ethanol injection (PEI) treatment is not currently a prevalent treatment option in Korea. PEI treatment may, however, be an alternative treatment option for locally recurrent thyroid carcinomas in properly selected patients [18].

Many Korean respondents may prefer high-dose RAI treatment because it is an important treatment modality for patients with recurrent papillary thyroid carcinoma in many institutions in Korea. The Korean respondents also preferred external irradiation more than did the JSTS or IAES respondents.

\section{Conclusions}

We found that actual treatment decisions for pa- tients in Korea with well differentiated thyroid carcinoma (WDTC) differed slightly from those in other countries. Among these differences were the extent of thyroid resection and lymph node dissection for papillary thyroid carcinoma smaller than $2 \mathrm{~cm}$, the dependence of radioactive iodine treatment on the extent of thyroidectomy, the indication and duration of TSH suppression therapy, and modalities for recurrent papillary thyroid carcinoma. The Korean respondents favored more aggressive surgical and postoperative RAI treatment than did the respondents from the other countries evaluated.

\section{Acknowledgements}

We would like to express our sincere appreciation to the members of the Korean Association of Endocrine Surgeons (KAES) who took the time to respond to our questionnaires despite their busy schedules.

\section{References}

1. Shigematsu N, Takami H, Ito N, Kubo A (2005) Nationwide survey on the treatment policy for well-differentiated thyroid cancer-Results of a questionnaire distributed at the 37 th meeting on the Japanese Society of Thyroid Surgery. Endocr J 52: 479-491.

2. Shigematsu N, Takami H, Kubo A (2006) Unique treatment policy for well-differentiated thyroid cancer in Japan: results of a questionnaire distributed to members of the Japanese Society of Thyroid Surgery and the International Association of Endocrine Surgeons. Endocr J 53: 829-839.

3. Ito $\mathrm{Y}$, Tomoda $\mathrm{C}$, Uruno $\mathrm{T}$, Takamura $\mathrm{Y}$, Miya A, Kobayashi K, Matsuzaka F, Kuma K, Miyauchi A (2004) Papillary microcarcinoma of the thyroid: how should it be treated? World J Surg 28: 1115-1121.

4. Cooper DS, Doherty GM, Haugen BR, Kloos RT, Lee SL, Mandel SJ, Mazzaferri EL, McIver B, Sherman SI, Tuttle RM; The Americal Thyroid Association Guidelines Taskforce (2006) Management guidelines for patients with thyroid nodules and differentiated thyroid cancer. Thyroid 16: 109-142.

5. Hagag P, Strauss S, Weiss M (1998) Role of ultrasound-guided fine needle aspiration biopsy in evaluation of nonpalpable thyroid nodules. Thyroid 8: 989 995.

6. Lin JD, Chao TC, Huang BY, Chen ST, Chang HY,
Hsueh C (2005) Thyroid cancer in the thyroid nodules evaluated by ultrasonography and fine-needle aspiration cytology. Thyroid 15: 708-717.

7. National Comprehensive Cancer Network: Thyroid carcinoma, Clinical practice guidelines in oncologyv.1.2994. http://www.nccn.org/professionals/physician gls/f_guidelines.asp, 2004.

8. Taylor T, Specker B, Robbins J, Sperling M, Ho M, Ain K, Bigos ST, Brierley J, Cooper D, Haugen B, Hay I, Hertzberg V, Klein I, Klein H, Ladenson P, Nishiyama R, Ross D, Sherman S, Maxon HR (1998) Outcome after treatment of high-risk papillary and non-Hurthle-cell follicular thyroid carcinoma. Ann Intern Med 129: 622-627.

9. Robbins RJ, Schlumberger M (2005) The evolving role of I-131 for the treatment of differentiated thyroid cancer. J Nucl Med 46: 28S-37S.

10. Durante C, Haddy N, Baudin E, Leboulleux S, Hartl D, Travagil JP, Caillou B, Ricard M, Lumbroso JD, De Vathaire F, Schlumberger M (2006) Long-term outcome of 444 patients with distant metastases from papillary and follicular thyroid carcinoma: Benefits and limits of radioiodine therapy. J Clin Endocrinol Metab 91: 2892-2899.

11. Maxon HR 3rd, Smith HS (1990) Radioiodine-131 in the diagnosis and treatment of metastatic well differen- 
tiated thyroid cancer. Endocrinol Metab Clin North Am 19: 685-718.

12. Mazzaferri EL, Jhiang SM (1994) Long-term impact of initial surgical and medical therapy on papillary and follicular thyroid cancer. Am J Med 97: 418-428.

13. McGriff NJ, Csako G, Gourgiotis L, Lori CG, Pucino F, Sarlis NJ (2002) Effects of thyroid hormone suppression therapy on adverse clinical outcomes in thyroid cancer. Ann Med 34: 554-564.

14. Vickery AL Jr, Wang CA, Walker AM (1987) Treatment of intrathyroidal papillary carcinoma of the thyroid. Cancer 60: 2587-2595.

15. Haugen B, Pacini F, Reiners C, Schlumnerger M, Ladenson PW, Sherman SI, Cooper DS, Graham KE, Braverman LE, Skarulis MC, Davies TF, DeGroot LJ, Mazzaferri EL, Daniels GH, Ross DS, Luster M, Samuels MH, Becker DV, Maxon HR 3rd, Cavalieri RR, Spencer CA, McEllin K, Weintraub BD, Ridgway EC (1999) A comparison of recombinant human thyrotropin and thyroid hormone withdrawal for the detection of thyroid remnant or cancer. J Clin Endocrinol Metab 84: 3877-3885.

16. Barbaro D, Boni G, Meucci G, Simi U, Lapi P, Orsini P, Pasquini G, Piazza F, Caciagli M, Mariani G (2003) Radioiodine treatment with $30 \mathrm{mCi}$ after recombinant human thyrotropin stimulation in thyroid cancer: effectiveness for postsurgical remnants ablation and possible role of iodine content in L-thyroxine in the outcome of ablation. J Clin Endocrinol Metab 88: 4110-4115.

17. Pacini F, Lari R, Mazzeo S, Grasso L, Taddei D, Pinchera A (1985) Diagnostic value of a single serum thyroglobulin determination on and off thyroid suppressive therapy in the follow-up of patients with differentiated thyroid cancer. Clin Endocrinol 23: 405411.

18. Lim CY, Yun JS, Lee J, Nam KH, Chung WY, Park CS (2007) Percutaneous ethanol injection therapy for locally recurrent papillary thyroid carcinoma. Thyroid 17: $347-350$. 